\title{
Increased Serum Neurofilament Light and Thin Ganglion Cell-Inner Plexiform Layer Are Additive Risk Factors for Disease Activity in Early Multiple Sclerosis
}

Ting-Yi Lin, MD, Viktoriya Vitkova, MSc, Susanna Asseyer, MD, Ivette Martorell Serra, MSc, Seyedamirhosein Motamedi, PhD, Claudia Chien, PhD, Marc Ditzhaus, PhD, Athina Papadopoulou, MD, Pascal Benkert, PhD, Jens Kuhle, MD, Judith Bellmann-Strobl, MD, Klemens Ruprecht, MD, Friedemann Paul, MD, Alexander U. Brandt, MD, and Hanna G. Zimmermann, PhD

Neurol Neuroimmunol Neuroinflamm 2021;8:e1051. doi:10.1212/NXI.0000000000001051

\section{Abstract}

\section{Objective}

To investigate the association of combined serum neurofilament light chain (sNfL) and retinal optical coherence tomography (OCT) measurements with future disease activity in patients with early multiple sclerosis (MS).

\section{Methods}

We analyzed sNfL by single molecule array technology and performed OCT measurements in a prospective cohort of 78 patients with clinically isolated syndrome and early relapsing-remitting MS with a median (interquartile range) follow-up of 23.9 (23.3-24.7) months. Patients were grouped into those with abnormal or normal sNfL levels, defined as $\mathrm{sNfL} \geq /<80$ th percentile of age-corrected reference values. Likewise, patients were grouped by a median split into those with thin or thick ganglion cell and inner plexiform layer (GCIP), peripapillary retinal nerve fiber layer, and inner nuclear layer in nonoptic neuritis eyes. Outcome parameters were violation of no evidence of disease activity (NEDA-3) criteria or its components.

\section{Results}

Patients with abnormal baseline sNfL had a higher risk of violating NEDA-3 (hazard ratio [HR] 2.28, 95\% CI 1.27-4.09, $p=0.006$ ) and developing a new brain lesion (HR 2.47, 95\% CI $1.30-4.69, p=0.006$ ), but not for a new relapse (HR 2.21, 95\% CI 0.97-5.03, p = 0.058). Patients with both abnormal sNfL and thin GCIP had an even higher risk for NEDA-3 violation (HR 3.61, 95\% CI 1.77-7.36, $p=4.2 \mathrm{e}^{-4}$ ), new brain lesion (HR 3.19, 95\% CI 1.51-6.76, $p=$ 0.002 ), and new relapse (HR 5.38, 95\% CI 1.61-17.98, $p=0.006$ ) than patients with abnormal sNfL alone.

\section{Conclusions}

In patients with early MS, the presence of both abnormal sNfL and thin GCIP is a stronger risk factor for future disease activity than the presence of each parameter alone.

\author{
Correspondence \\ Dr. Zimmermann \\ hanna.zimmermann@charite.de
}

\section{RELATED ARTICLE}

Editorial

Combined Neurofilament

Light and Optical

Coherence Tomography

Better Predicts Multiple

Sclerosis Disease Activity

Than Either Measure

Alone

Page e1054 


\section{Glossary}

3D = 3-dimensional; ART = automatic real-time; CIS = clinically isolated syndrome; DMT = disease-modifying therapy; EDSS = Expanded Disability Status Scale; FLAIR = fluid-attenuated inversion recovery; GCIP = ganglion cell and inner plexiform layer; Gd+ = gadolinium-enhancing; HeyEx = Heidelberg Eye Explorer; HR = hazard ratio; INL = inner nuclear layer; IQR = interquartile range; LMM = linear mixed effect model; MPRAGE = magnetization prepared rapid acquisition gradient echo; MS = multiple sclerosis; NEDA-3 = no evidence of disease activity; NfL = neurofilament light chain; OCT = optical coherence tomography; $\mathbf{O N}$ = optic neuritis; $\mathbf{p R N F L}$ = peripapillary retinal nerve fiber layer; RRMS = relapsing-remitting MS; $\mathbf{s N f L}=$ serum NfL; T2w $=$ T2-weighted; TE $=$ echo time; $\mathbf{T I}=$ inversion time; $\mathbf{T R}=$ repetition time.

Multiple sclerosis (MS) usually manifests with a monophasic demyelinating episode, referred to as clinically isolated syndrome (CIS). ${ }^{1,2}$ As the further clinical course of MS can be highly variable, ${ }^{3}$ reliable biomarkers for future disease activity in patients at early stage of the disease are valuable for individual counseling and guiding treatment decisions.

Optical coherence tomography (OCT) is a noninvasive technique that can visualize axonal loss (peripapillary retinal nerve fiber layer [pRNFL] thinning), neuronal damage (ganglion cell and inner plexiform layer [GCIP] thinning), and neuroinflammation (inner nuclear layer [INL] edema) in the retina. ${ }^{4-7}$ Our previous work showed that in patients with CIS, reduced GCIP thickness is associated with future disease activity. ${ }^{8}$

Neurofilament light chain (NfL) protein is a promising biomarker for neuroaxonal damage. ${ }^{9,10}$ Serum NfL (sNfL) levels in MS were found to increase in cases where MRI measurements indicated new or enlarged brain lesions and decreased brain volume and were associated with diffuse white matter damage. ${ }^{9,11-14}$ In addition, higher sNfL levels in patients with early MS were also associated with higher probability of disability worsening. ${ }^{15}$

Despite the fact that retinal layer thinning is a chronic process, and sNfL reflects shorter-term disease activity, ${ }^{9,16}$ both parameters may each provide a value for subsequent disease activity. However, the combined association of elevated sNfL and abnormal OCT measurements with future disease activity in patients with CIS/early MS has not been well-explored.

In this study, we aimed at investigating the potentially additive value of sNfL and OCT measurements as markers for subsequent disease activity in patients with CIS or early MS.

\section{Methods}

\section{Study Design}

All patients included in this study participate in an ongoing prospective, observational cohort study (Berlin CIS cohort: ClinicalTrials.gov identifier: NCT01371071) at the NeuroCure Clinical Research Center, Charité-Universitätsmedizin Berlin of patients with a CIS or early MS (up to 2 years since disease onset) according to the McDonald 2017 criteria. $^{2}$ The baseline OCT measurements and sNfL samples in this study were collected at the same visit. All patients underwent annual follow-up visits for clinical examination, serum sample collection, retinal OCT, and brain MRI scanning.

Inclusion criteria for this study were (1) the availability of baseline serum samples obtained between 12 and 24 months after the first disease manifestation and (2) a follow-up period of at least 12 months after enrollment. Exclusion criteria were (1) relapse events within 120 days before the study inclusion, (2) optic neuritis $(\mathrm{ON})$ in both eyes, or (3) contraindications for OCT analysis, including a refractive error above $\pm 6 \mathrm{di}$ opters or retinopathy unrelated to MS.

The primary study outcome was violating the no evidence of disease activity (NEDA-3) criteria, which are composed of the absence of new relapses, no new gadolinium-enhancing $(\mathrm{Gd}+)$ or T2-hyperintense lesions on MRI, and the absence of confirmed Expanded Disability Status Scale (EDSS) worsening. ${ }^{17}$ We also analyzed the 3 components of NEDA-3 as separate outcome parameters. Confirmed EDSS worsening was defined as an increase of at least 1.5, 1.0, or 0.5 and consequently being sustained in the consecutive annual follow-up visit in patients with a baseline EDSS score of 0, at least 1.0 or above 5.5 , respectively. ${ }^{18}$

\section{Standard Protocol Approvals, Registrations, and Patient Consents}

This study followed the Declaration of Helsinki in its currently applicable version and was approved by the institutional ethics committee at the Charité-Universitätsmedizin Berlin (EA1/ 182/10). All participants provided written informed consent before their inclusion in the study.

\section{sNfL Measurements}

sNfL was measured at the Department of Neurology, University of Basel, Switzerland, in frozen serum samples shipped on dry ice. Operators masked to clinical data performed the analysis using a highly sensitive single molecule array (Simoa Technology; QUANTERIX Corporation, Billerica, MA) immunoassay. ${ }^{11,19}$

\section{Optical Coherence Tomography}

All OCT images were obtained under normal room light conditions by experienced operators through spectral domain OCT and Heidelberg Eye Explorer (HeyEx) version 1.9.10.0 
(Heidelberg Engineering, Heidelberg, Germany) with automatic real-time (ART) function for image averaging and an activated eye tracker. All individual scans were quality controlled according to the OSCAR-IB criteria ${ }^{20,21}$ and reported based on the APOSTEL recommendations. ${ }^{22}$ A peripapillary circular scan within a $12^{\circ}(\sim 3.4 \mathrm{~mm})$ diameter ring $(1,536$ A-scans; $9 \leq \mathrm{ART} \leq 99$ ) around the optic nerve head was used to measure the pRNFL thickness. The pRNFL on ring scans was segmented with HeyEx viewing module version 6.0.14.0. Macular volume scans $\left(25^{\circ} \times 30^{\circ}\right.$, 61 vertical B-scans, $12 \leq$ ART $\leq 18$ ) were segmented with the SAMIRIX pipeline. ${ }^{23}$ The GCIP and INL thickness were calculated as a $6-\mathrm{mm}$ diameter cylinder around the fovea. ${ }^{24}$ Segmentation errors were manually corrected if necessary.

\section{MRI}

All MRI data were acquired using two $3 \mathrm{~T}$ machines (Tim Trio; Siemens, Erlangen, Germany). The scanning protocol included a 3-dimensional (3D) T1-weighted magnetization prepared rapid acquisition gradient echo (MPRAGE) sequence (repetition time $[\mathrm{TR}]=1,900$ milliseconds, echo time $[\mathrm{TE}]=2.55$ milliseconds, inversion time $[\mathrm{TI}]=900$ milliseconds) for precontrast and postcontrast administration and 3D T2-weighted $(\mathrm{T} 2 \mathrm{w})$ fluid-attenuated inversion recovery (FLAIR) sequence $(\mathrm{TR} / \mathrm{TE} / \mathrm{TI}=6,000 / 388 / 2,100$ milliseconds; $1 \times 1 \times 1 \mathrm{~mm}^{3}$ resolution).

For brain lesion segmentation, precontrast and postcontrast MPRAGE images were coregistered to MNI-152 standard space for all patients using FMRIB's Linear Image Registration Tool from FMRIB Software Library with linear-rigid registration. ${ }^{25,26}$ Subsequently, FLAIR images were coregistered with precontrast MPRAGE images. Brain T2hyperintense lesions were manually marked and segmented using both FLAIR and MPRAGE scans to create binary masks by 2 expert raters under the supervision of a board-certified radiologist using ITK-SNAP (itksnap.org). ${ }^{27} \mathrm{Gd}+$ lesions were segmented by the same raters and ITK-SNAP using postcontrast MPRAGE scans only.

\section{Statistical Analysis}

Statistical analysis was performed in $\mathrm{R}$ version 4.0.2 with packages lme4, lmerTest, MuMIn, ggplot2, ggpubr, survival, and survminer. ${ }^{28}$ Continuous variables are presented as either mean with SD or median with interquartile range (IQR).

Only OCT measurements of eyes with no history of $\mathrm{ON}$ at baseline were included. GCIP was considered the primary OCT variable because it has shown highest associations with future disease activity in an earlier study, ${ }^{8}$ whereas pRNFL and INL were analyzed as exploratory variables.

Age-specific sNfL percentile values were calculated from a generalized additive models for location scale and shape model based on a large cohort of 259 healthy controls provided by the Department of Neurology, University of Basel, Switzerland. ${ }^{9}$ Under the concern of potential ceiling effect while using percentiles, age-specific sNfL Z-scores were also derived from the same model.

The baseline associations of sNfL and OCT measurements including pRNFL, GCIP, and INL thickness were analyzed using linear mixed effect model (LMM) (fixed effect: sNfL value; random effects: inter-eye within-patient correlations). No imputation was carried out in the analysis.

To investigate the risk of violating the NEDA-3 criteria or its components with sNfL or/and OCT parameters as risk factors, Kaplan-Meier analyses and Cox regression models were implemented. In case of measurements for 2 non-ON eyes were available, the mean of both eyes was used. After performing sensitivity analysis (eTable 1, links.lww.com/NXI/ A523, and eTable 2, links.lww.com/NXI/A524), we defined sNfL values higher than the 80th percentile as abnormal sNfL and used median as dividers for each OCT parameter. Thinner pRNFL and GCIP and thicker INL were considered as pathologic, respectively. To assess the individual risk associated with a combination of sNfL and OCT groups, we combined the sNfL and OCT parameters together as 1 variable with 4 categories: (1) normal sNfL + thick GCIP/thick pRNFL/thin INL, (2) normal sNfL + thin GCIP/thin pRNFL/thick INL, (3) abnormal sNfL + thick GCIP/thick pRNFL/thin INL, and (4) abnormal sNfL + thin GCIP/thin pRNFL/thick INL. In addition, we computed multivariable Cox regression models including-besides $\mathrm{sNfL}$ and GCIP thickness-age, sex, T2w lesion counts, and diseasemodifying therapy (DMT), which are previously established factors associated with future disease activity in patients with CIS/early MS. ${ }^{29}$ Analysis was censored after 760 days because of low numbers at risk after this period. Furthermore, we evaluated the interaction term between sNfL and GCIP thickness in the Cox regression models and the synergistic effects between the 2 factors by calculating the attributable proportion that was because of their interaction as a measure of excess risk. ${ }^{30}$

\section{Data Availability}

All data are available upon reasonable request from the corresponding author.

\section{Results}

\section{Cohort Characteristics}

Of 131 patients with CIS or early MS screened, 78 patients met all the inclusion criteria. Eight patients had baseline sNfL value acquired more than 24 months after the initial attack; 29 patients did not have follow-up of more than 365 days; 10 patients had a relapse within 120 days before the baseline sNfL collection; 2 patients had ON in both eyes; 4 patients had OCT measurements with insufficient quality in both eyes (eFigure 1, links.lww.com/NXI/A518). ${ }^{20,21}$

Among the 78 patients included, 2 pRNFL measurements from 2 patients and 5 GCIP and INL measurements from 5 other patients with insufficient OCT quality in 1 eye were 
omitted from the respective analyses. Sixty-two (79\%) patients fulfilled the $2017 \mathrm{McD}$ onald MS diagnostic criteria for relapsingremitting MS (RRMS) ${ }^{2}$; the remaining 16 (21\%) patients were considered CIS. Eighteen (23\%) patients with RRMS had clinically definite MS, defined by a second clinical episode. Demographic and clinical characteristics of the study cohort are summarized in Table 1 , and comorbidities are described in supplemental data (eTable 3, links.lww.com/NXI/A525).

\section{Baseline Associations}

LMM revealed an association between baseline age-specific sNfL Z-score with GCIP (B [SE] $=-0.921[0.448] \mu \mathrm{m}, p=$ 0.043 ), indicating that patients with $1 \mathrm{SD}$ higher in sNfL Z-score had a lower GCIP thickness by $0.921 \mu \mathrm{m}$. No association between baseline sNfL Z-score with pRNFL (B [SE] $=-1.495$ $[0.795] \mu \mathrm{m}, p=0.064)$ or INL (B $[\mathrm{SE}]=-0.156[0.168] \mu \mathrm{m}, p=$ 0.356) was found (eFigure 2, links.lww.com/NXI/A519).

\section{Association of sNfL and Retinal Thickness Measurements With Future Disease Activity}

Of the 78 patients included in this study, 46 (59\%) patients violated NEDA-3, 23 (29\%) patients experienced a clinical relapse, 38 (49\%) patients developed new T2w or Gd+ brain lesions on MRI, and 9 (12\%) patients had confirmed EDSS worsening during a median follow-up of 23.9 months. We first investigated baseline abnormal sNfL ( $\geq 80$ th reference percentile), thinner pRNFL ( $\leq 100 \mu \mathrm{m}$; median divider), thinner GCIP ( $\leq 70.4 \mu \mathrm{m}$; median divider), and thicker INL ( $\geq 36.4 \mu \mathrm{m}$; median divider) separately regarding an association with the risk for future violation of the NEDA-3 criteria.

Patients with baseline abnormal sNfL showed a higher risk of the NEDA-3 violation (hazard ratio [HR] 2.28, 95\% CI

Table 1 Baseline Cohort Characteristics

\begin{tabular}{ll}
\hline & Patients (N = 78) \\
\hline Sex, F (\%)/M (\%) & $50(64.1) / 28(35.9)$ \\
\hline Age, y, mean (SD) & $33.7(7.4)$ \\
\hline sNfL level, pg/mL, median (IQR) & $19.7(15.2-28.8)$ \\
\hline Time since disease onset, mo, median (IQR) & $12.1(11.8-12.7)$ \\
\hline Follow-up duration, mo, median (IQR) & $23.9(23.3-24.7)$ \\
\hline GCIP thickness, $\boldsymbol{\mu m}$, mean (SD) & $69.4(7.1)$ \\
\hline pRNFL thickness, $\boldsymbol{\mu m}, \mathbf{m e a n}(\mathrm{SD})$ & $98.7(12.9)$ \\
\hline INL thickness, $\boldsymbol{\mu m}, \mathbf{m e a n}(\mathrm{SD})$ & $36.8(2.3)$ \\
\hline Total T2w lesion count, N, median (IQR) & $11(5-28)$ \\
\hline Total T2w lesion volume, mL, median (IQR) & $1.1(0.4-2.8)$ \\
\hline EDSS score, median (IQR) & $1.5(1.0-2.0)$ \\
\hline
\end{tabular}

Abbreviations: EDSS = expanded disability status scale; GCIP = ganglion cell and inner plexiform layer; $I N L=$ inner nuclear layer; $I Q R=$ interquartile range; $\mathrm{pRNFL}=$ peripapillary retinal nerve fiber layer; $\mathrm{sNfL}=$ serum neurofilament light chain; $\mathrm{T} 2 \mathrm{w}=\mathrm{T} 2$-weighted.
1.27-4.09, $p=0.006$; Figure 1A), whereas thinner GCIP (Figure 1D), thinner pRNFL (eFigure 3A, links.lww.com/ NXI/A520), or thicker INL (eFigure 3D) showed no significant association with future NEDA-3 violation. When analyzing the association of sNfL with single components of NEDA-3, patients with abnormal sNfL had an increased risk of developing new lesions (HR 2.47, 95\% CI 1.30-4.69, $p=$ 0.006; Figure 1C), but not for a new relapse (HR 2.21, 95\% CI $0.97-5.03, p=0.058$; Figure 1B). Thinner GCIP was associated with a higher risk of a new relapse (HR 2.88, 95\% CI $1.18-7.01, p=0.021$; Figure $1 \mathrm{E}$ ), but not for new lesions. Neither abnormal sNfL nor OCT parameters showed an association with future confirmed EDSS worsening (results not shown). These results were also confirmed when taking OCT measurements as continuous variables instead of dichotomizing into categorical variables (eTable 4, links.lww. com/NXI/A526).

In the multivariable analysis, abnormal sNfL was associated with the NEDA-3 violation (HR 2.11, 95\% CI 1.16-3.86, $p=$ 0.015 ) and a new lesion (HR 2.04, 95\% CI 1.06-3.94, $p=$ 0.033 ), whereas higher $\mathrm{T} 2 \mathrm{w}$ lesion counts were associated with future lesion development (HR 1.01, 95\% CI 1.00-1.03, $p=$ 0.043). Patients with thinner GCIP had a higher, yet nonsignificant risk of new clinical relapse compared with patients with thicker GCIP. The risk of violating the NEDA-3 criteria was not affected by age, sex, or receiving DMT at the time of study enrollment (Table 2). Higher T2w lesion volume was also associated with subsequent NEDA-3 violation and lesion development (eTable 5, links.lww.com/NXI/A527).

To analyze whether sNfL and OCT parameters combined show stronger associations with future MS disease activity than each parameter alone, we combined sNfL and different OCT parameters as an univariable risk factor for further analysis. For the NEDA-3 criteria violation, patients with (1) abnormal sNfL + thin GCIP (HR 3.61, 95\% CI 1.77-7.36, $p=$ $4.2 \mathrm{e}^{-4}$; Figure $2 \mathrm{~A}$ ), (2) abnormal sNfL + thin pRNFL (HR 2.63, 95\% CI 1.21-5.70, $p=0.015$; eFigure 4A, links.lww. com/NXI/A521), or (3) abnormal sNfL + thick INL (HR 3.05, 95\% CI 1.32-7.05, $p=0.009$; eFigure 4D), all have increased risks in comparison with patients without abnormal sNfL or thin GCIP/thin pRNFL/thick INL as risk factors. Higher hazard ratios were observed when the sNfL and OCT measurements were combined than abnormal sNfL or thin GCIP/thin pRNFL/thick INL alone as a single risk factor, as in the analysis above. Regarding the occurrence of new relapses, the Cox regression analysis revealed a higher risk associated with (1) abnormal sNfL + thin GCIP (HR 5.38, 95\% CI 1.61-17.98, $p=0.006$; Figure 2B), (2) abnormal sNfL + thin pRNFL (HR 4.77, 95\% CI 1.39-16.38, $p=0.013$; eFigure 4B), or (3) abnormal sNfL + thick INL (HR 3.26, 95\% CI 1.09-9.76, $p=0.034$; eFigure $4 \mathrm{E}$ ) as a combined univariable risk factor. For new lesion development, an increased HR was achieved when combining abnormal baseline $\mathrm{sNfL}$ value with reduced GCIP thickness (HR 3.19, 95\% CI 1.51-6.76, $p=$ 0.002; Figure $2 \mathrm{C}$ ). As for confirmed worsening of the EDSS 

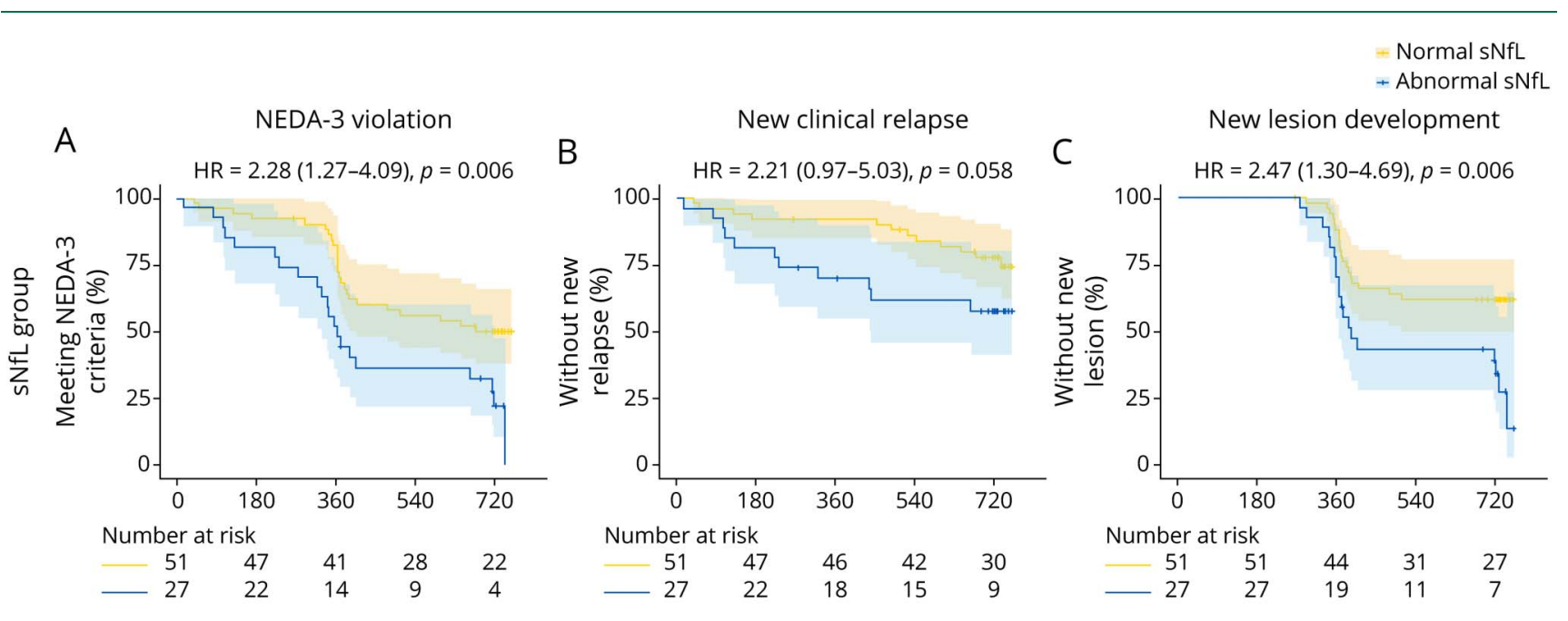

- Abnormal sNfL
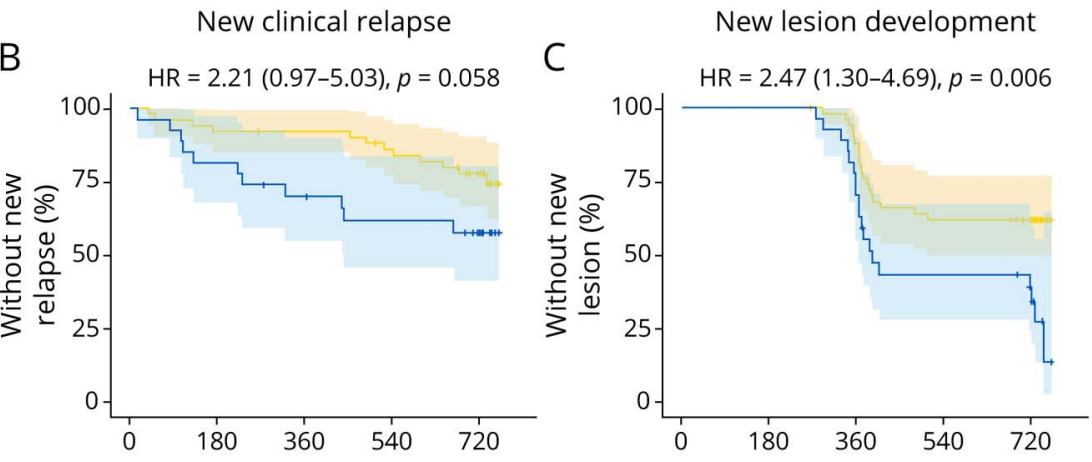

\section{Number at risk}

$\begin{array}{rrrrc}51 & 47 & 46 & 42 & 30 \\ -27 & 22 & 18 & 15 & 9\end{array}$

Number at risk

$\begin{array}{rrrrr}51 & 51 & 44 & 31 & 27 \\ -27 & 27 & 19 & 11 & 7\end{array}$

$\mathrm{D}$

E

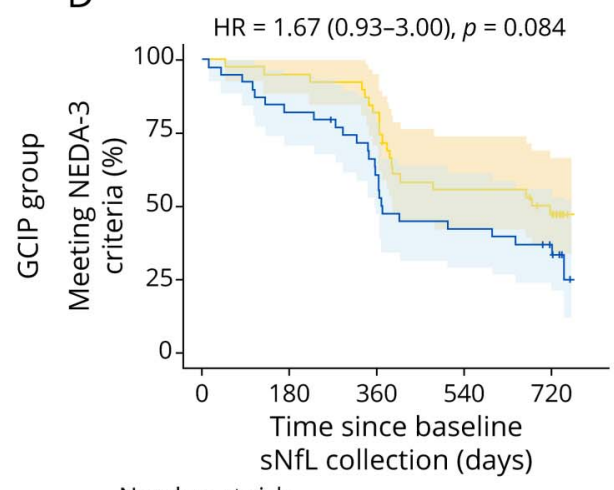
Number at risk NfL collection (days)

$\begin{array}{rrrrr}39 & 37 & 32 & 21 & 16 \\ -39 & 32 & 23 & 16 & 10\end{array}$

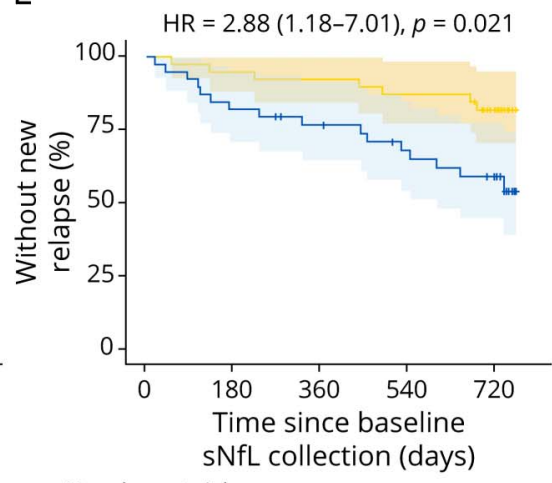
Number at risk

$\begin{array}{rrrrr}39 & 37 & 36 & 34 & 23 \\ -39 & 32 & 28 & 23 & 16\end{array}$

$\mathrm{F}$

$=$ Thick GCIP

+ Thin GCIP

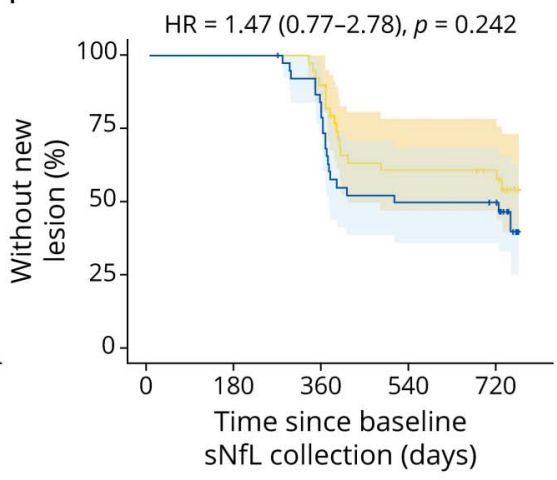

Number at risk

\begin{tabular}{rrrrr}
39 & 39 & 35 & 23 & 19 \\
-39 & 39 & 28 & 19 & 15 \\
\hline
\end{tabular}

Patients violating NEDA-3 criteria with (A) abnormal sNfL or (D) thin GCIP as risk factors, patients experiencing new relapse with (B) abnormal sNfL or (E) thin GCIP as risk factors, and patients having new lesion development with (C) abnormal sNfL or (F) thin GCIP as risk factors. Shady regions describe the $95 \%$ Cl of the curve. GCIP = ganglion cell and inner plexiform layer; HR = hazard ratio; NEDA-3 = no evidence of disease activity-3 criteria; sNfL = serum neurofilament light chain.

Table 2 Multivariable Analysis With Cox Proportional Hazard Models of Factors Associated With NEDA-3 Violation, Relapse, and Lesion Development

\begin{tabular}{|c|c|c|c|c|c|c|}
\hline & \multicolumn{2}{|l|}{ NEDA-3 violation } & \multicolumn{2}{|l|}{ Relapse } & \multicolumn{2}{|l|}{ New lesion } \\
\hline & $\mathrm{HR}(95 \% \mathrm{Cl})$ & $p$ Value & HR $(95 \% \mathrm{Cl})$ & $p$ Value & HR $(95 \% \mathrm{Cl})$ & $p$ Value \\
\hline sNfL $\geq 80$ th percentile & $2.11(1.16-3.86)$ & $0.015^{\mathrm{a}}$ & $1.98(0.85-4.64)$ & 0.114 & $2.04(1.06-3.94)$ & $0.033^{\mathrm{a}}$ \\
\hline GCIP $\leq 70.4 \mu \mathrm{m}$ & $1.42(0.78-2.60)$ & 0.254 & $2.42(0.97-6.03)$ & 0.057 & $1.14(0.58-2.26)$ & 0.707 \\
\hline Age & $1.00(0.96-1.04)$ & 0.864 & $0.99(0.94-1.05)$ & 0.746 & $1.00(0.96-1.04)$ & 0.815 \\
\hline Sex, male & $1.39(0.75-2.61)$ & 0.299 & $1.51(0.63-3.61)$ & 0.355 & $0.89(0.44-1.80)$ & 0.745 \\
\hline T2w lesion count & $1.01(1.00-1.02)$ & 0.081 & $1.01(0.99-1.02)$ & 0.531 & $1.01(1.00-1.03)$ & $0.043^{a}$ \\
\hline DMT at baseline & $1.53(0.82-2.84)$ & 0.180 & $1.71(0.68-4.26)$ & 0.252 & $1.20(0.61-2.36)$ & 0.601 \\
\hline
\end{tabular}

Abbreviations: DMT = disease-modifying therapy; GCIP = ganglion cell and inner plexiform layer; $\mathrm{HR}=$ hazard ratio; NEDA-3 = no evidence of disease activity-3 criteria; $\mathrm{SNfL}=$ serum neurofilament light chain; $\mathrm{T} 2 \mathrm{w}=\mathrm{T} 2$-weighted.

a Significant. 
Figure 2 Kaplan-Meier Plots of Meeting NEDA-3 Criteria or Its Components With Abnormal sNfL and OCT Measurements as Combined Risk Factor

A. NEDA-3 violation

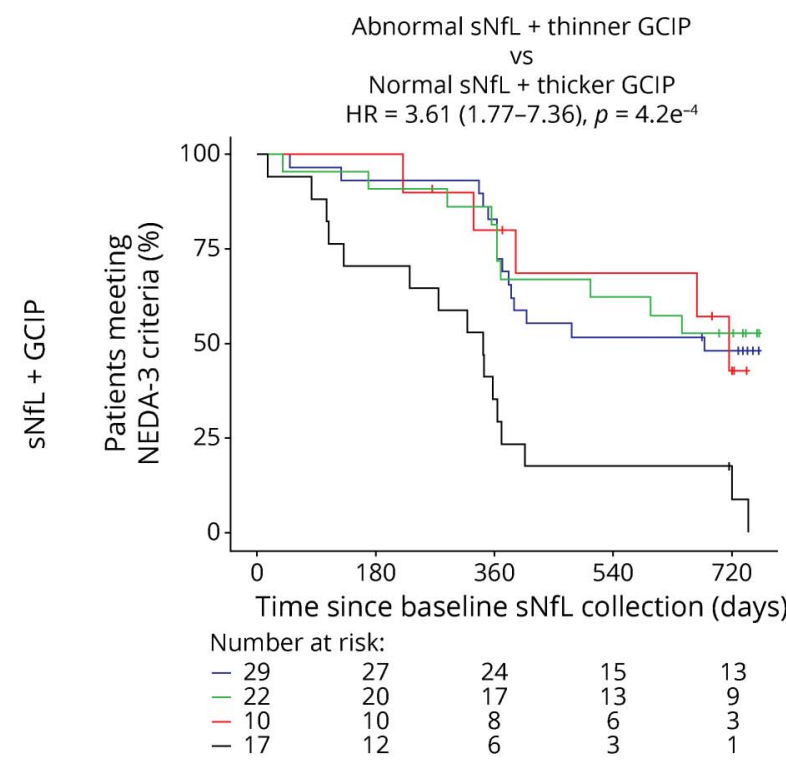

C. New lesion development

Abnormal sNfL + thinner GCIP

vs

Normal sNfL + thicker GCIP

$\mathrm{HR}=3.19(1.51-6.76), p=0.002$

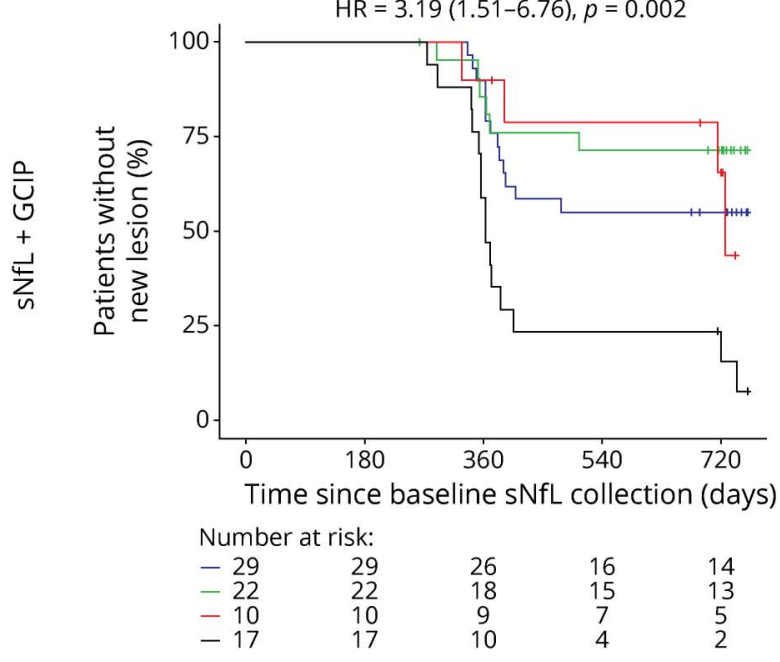

B. New clinical relapse

Abnormal sNfL + thinner GCIP vs

Normal sNfL + thicker GCIP $H R=5.38(1.61-17.98), p=0.006$

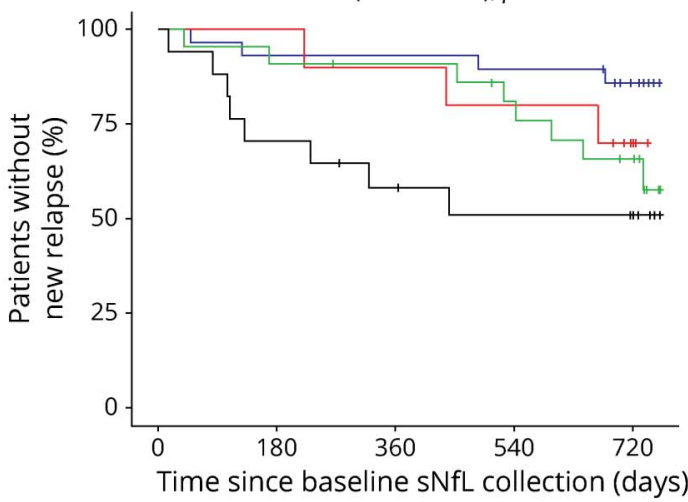

Number at risk:

$\begin{array}{ccccc}-29 & 27 & 27 & 26 & 19 \\ -22 & 20 & 19 & 16 & 11 \\ -10 & 10 & 9 & 8 & 4 \\ -17 & 12 & 9 & 7 & 5\end{array}$

+ Normal sNfL + thick GCIP

+ Normal sNfL + thin GCIP

+ Abnormal sNfL + thick GCIP

+ Abnormal sNfL + thin GCIP

Cumulative risk of patients with abnormal sNfL value and thin GCIP (black), abnormal sNfL value and thick GCIP (red), normal sNfL value and thin GCIP (green), and normal sNfL value and thick GCIP (blue) for (A) NEDA-3 violation; (B) relapse; and (C) a new lesion. GCIP = ganglion cell and inner plexiform layer; HR = hazard ratio; NEDA-3 = no evidence of disease activity-3 criteria; OCT = optical coherence tomography; sNfL = serum neurofilament light chain.

score, a higher association cannot be achieved in combination of sNfL with any of the 3 OCT parameters (results not shown). A summary of the HRs for future disease activity outcomes associated with abnormal sNfL and thinner GCIP alone and their combination is presented in Figure 3.

The interaction between abnormal sNfL and thin GCIP is summarized in the supplemental data (eTable 6, links.lww. com/NXI/A528). We found an interaction effect between abnormal sNfL and thin GCIP for future NEDA-3 violation and new lesion development, but not for relapses. The attributable proportion showed that the interaction of abnormal sNfL and thin GCIP added $75 \%$ to the subsequent NEDA-3 violation risk and $85 \%$ to the new brain lesion risk determined with the sum of the individual risks (eTable 6).

\section{Discussion}

We investigated the relation between baseline sNfL levels and OCT and their individual and combined association with future disease activity in a well-characterized cohort of patients with CIS or early MS. We found (1) a weak cross- 


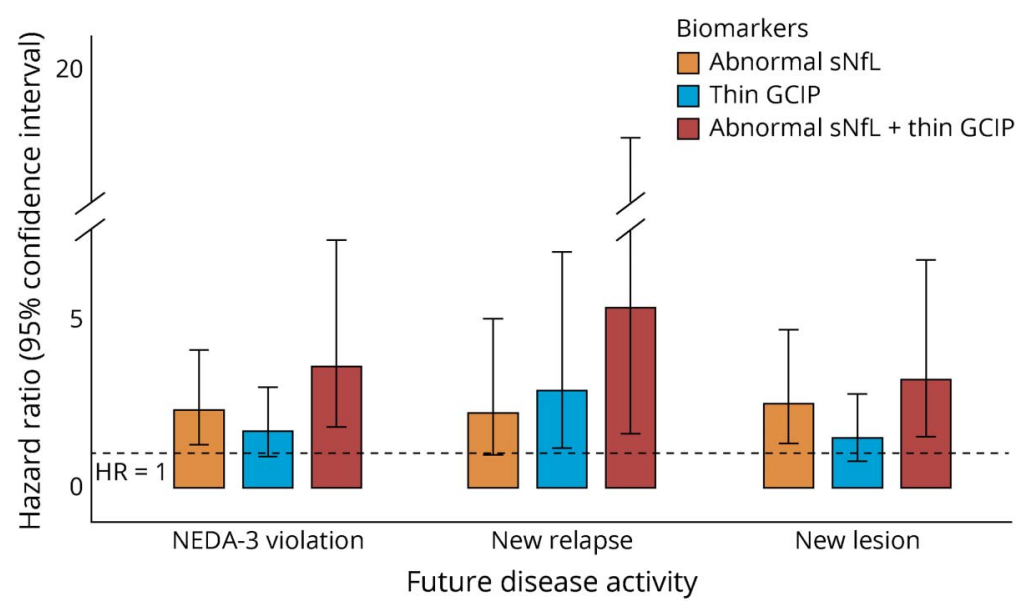

$\mathrm{GCIP}=$ ganglion cell and inner plexiform layer; $\mathrm{HR}=$ hazard ratio; NEDA-3 = no evidence of disease activity-3 criteria; sNfL = serum neurofilament light chain.

sectional association of increased sNfL levels with thinner GCIP but no association of sNfL with pRNFL or INL; (2) an association of increased sNfL with future NEDA-3 violation, mainly driven by the development of new lesions; (3) an association of thinner GCIP with future relapses; and (4) combinations of increased sNfL levels with thinner GCIP or pRNFL or thicker INL is associated with a higher risk of future disease activity than each parameter alone.

Two studies had previously investigated the relationship between sNfL and OCT values. ${ }^{31,32}$ These studies found contradictory results regarding cross-sectional associations of $\mathrm{sNfL}$ levels with retinal layer thickness measurements: The first studyreported results of 80 patients with RRMS from a 3-year study showed that an increase of sNfL level is associated with the annual reduction of pRNFL. ${ }^{31}$ However, no cross-sectional association of sNfL and pRNFL was identified. On the contrary, the second study reported cross-sectional evidence that sNfL concentration was significantly associated with both pRNFL and GCIP from 110 patients with MS. ${ }^{32}$ Our baseline results showed that the sNfL level is associated with GCIP but missed significance for an association with pRNFL. Both studies have only limited comparability with each other and to our study because they analyzed patients with RRMS with a mean disease duration of 4.6 years (SD 5.0) $)^{31}$ and a mixed RRMS and progressive MS cohort with a median disease duration of 16.5 years (IQR 8.7-23.0), ${ }^{32}$ respectively, whereas our study included patients with CIS and early MS with a median disease duration of 12.1 months (IQR 11.8-12.7). The association between increased sNfL levels and neuroaxonal retinal damage might be more pronounced in later and more progressive stages of the disease because it was the case in the cohort of the second study. ${ }^{32}$ However, this potentially may not result from a causal association but from a general and less focal chronic neurodegeneration.

In our study, abnormal sNfL levels were associated with an increased risk of subsequent violation of the NEDA-3 criteria. Regarding the components of the NEDA-3 criteria, abnormal
sNfL was especially related to a 2.5 -fold increased risk of new brain lesions development on MRI. As for the other 2 components in the NEDA-3 criteria contravention, we did not observe an association of abnormal sNfL with future relapses or confirmed EDSS worsening. Only 9 patients in our cohort had confirmed EDSS increase within the observation period, limiting the power of the latter analyses.

To determine whether the effects of sNfL were independently associated with future disease activity, several risk factors for MS disease activity were included for the multivariable analysis. Patients with more brain MRI lesions were identified to be a high-impact prognostic factor for future disease activity in large prospective cohorts previously. ${ }^{29,33}$ Nevertheless, our study demonstrated that patients with more T2w lesions on brain MRI had a higher but nonsignificant $H R$ for violating the NEDA-3 criteria. The resulting difference could be explained by the fact that our study only had a relatively small sample size and shorter follow-up duration compared with the previous studies. Furthermore, our cohort comprised patients with a higher lesion burden than in other studies, in which a large portion of patients did not show any lesion at all.

Our group has shown earlier that thinner GCIP and pRNFL are associated with future disease activity in CIS and early MS, in a cohort partly overlapping with this study. ${ }^{8}$ Interestingly, the association of thinner GCIP and pRNFL with future NEDA-3 violation was almost exclusively driven by an association with new lesions in the previous study; although in this study, it is mainly driven by an association with future relapses. This can be explained by (1) different patients-only 31 patients who overlap between this and the previous study, (2) different timepoints-the earlier study established the baseline visit in the first year after onset, whereas the baseline for this study is at the end of the first or second year after disease onset, (3) diagnostic criteria-the earlier study included patients with CIS in line with the $2010 \mathrm{McD}$ onald criteria; this study included patients with CIS and RRMS in 
line with the 2017 McDonald criteria, and (4) different protocols - this study has only annual MRI follow-up visits, whereas patients received more frequent follow-ups in the first year after onset, which was analyzed in the earlier study. Despite these differences, we would have expected a stronger association of thinner GCIP with future new lesions. An investigation of those associations in larger and independent cohorts would be helpful to unravel these inconsistencies.

The most important finding of our study is that thinning of GCIP and pRNFL can both provide combined benefits to abnormal sNfL regarding their association with subsequent NEDA-3 violation, as demonstrated by higher HRs. In addition, abnormal sNfL value with thinner pRNFL or GCIP shows a higher association with future relapses, and abnormal sNfL value with thinner GCIP shows a higher HR for a new lesion, in comparison to sNfL alone, respectively. The combined benefits of sNfL with either GCIP or pRNFL thickness may be explained by the nature of the biomarkers. The concentration of sNfL in samples collected within 3-4 months after relapse was significantly higher compared with the samples collected after this time window or during remission phase. ${ }^{9,16}$ In contrast with sNfL, both GCIP and pRNFL thickness on OCT show continuous thinning over time, reflecting a chronic neuroaxonal injury. ${ }^{6}$ Patients with MS who have both abnormal acute and chronic biomarkers could indicate that the damage is more profound, leading to a higher rate of NEDA-3 violation. Together, our data suggest a synergistic effect of GCIP/pRNFL and sNfL measurements and exemplifies the potential when combining biomarkers to increase disease relevance. NfL by itself is unspecific for MS-related damage as it is, e.g., increased in other neurodegenerative disorders ${ }^{34}$ and in association with cardiovascular risk factors or traumatic brain injury. ${ }^{35}$ The normal range of pRNFL and GCIP thickness is broad, impeding an individual risk assessment in early MS. ${ }^{8}$ But together, the combination of abnormal sNfL and thin GCIP/pRNFL may have the specificity to identify a group of patients in which both markers are related to MS-associated damage and thus outperforms the prognostic value of each marker alone.

INL thickening can mirror not only active retinal inflammation but the inflammatory status of MS disease activity. ${ }^{36}$ Longitudinal reduction in INL volume was found to be correlated with meeting the NEDA-3 criteria. ${ }^{37}$ Although, in our study, thicker INL alone was not a risk factor for future disease activity, we still demonstrate that thicker INL increases the hazard ratio of abnormal sNfL as a risk factor for NEDA-3 violation and new relapses. The thickening of INL in MS could be caused by dynamic change of fluid homeostasis in the retinal glymphatic system or dysfunction of the Müller cell. ${ }^{36}$ However, the true mechanism of INL thickening in patients with MS remains unclear. Therefore, although INL thickening and elevated sNfL level both suggest active inflammation in patients with MS, no established evidence has proven the causality between them.

The timing for retinal OCT examination and sNfL sample collection should be taken into consideration in clinical application. During acute episode, the pRNFL is often affected by early inflammation or edema within 3 months, whereas GCIP thickness seems to decrease. ${ }^{38-40}$ Afterward, both pRNFL and GCIP continue to have progressive thinning. ${ }^{39,40}$ Therefore, the quantification of retinal layer thickness should be made no earlier than 3 months after the attack. On the other hand, the concentration of sNfL will transiently increase during relapse ${ }^{11}$ and is likely to return to a steady state 4 months afterwards. ${ }^{9,16}$ Our study started with 12 months after first clinical event and excluded patients with clinical relapses within 120 days before the inclusion, presenting the utility of these 2 biomarkers under a disease stable phase.

Strengths of our study include its prospective design, standardized acquisition of clinical and paraclinical data, and a decent follow-up time. In addition, the dividers we chose for sNfL and OCT measurements are similar to previous studies, supporting the generalizability of our results. ${ }^{31,41}$ However, because only few patients showed confirmed EDSS worsening during the follow-up period, we could not adequately address the influence of sNfL and OCT parameters on EDSS worsening. The limited sample size may also mask significant effects of age, sex, or treatment in the multiple Cox regression model. In addition, our study lacks the power to determine the optimal cutoff value for either sNfL or OCT parameters through receiver operator characteristic analyses, limiting the generalizability of our results. Furthermore, there are several factors that may cause within-subject sNfL variation. ${ }^{42-45} \mathrm{Al}-$ though none of the patients in our cohort have small infarcts or neuropathy, we did not adjust the analysis for body mass index, participation in physical contact sports, high systolic blood pressure, and hemoglobin Alc level. Larger-scope multicenter studies should consider tackling these limitations to establish more concrete evidence on the relationship between $\mathrm{SNfL}$ and different OCT measurements throughout the disease course.

In conclusion, our study demonstrates an additive, potentially synergistic, effect of sNfL and retinal OCT, especially GCIP, as risk factors for future disease activity in patients with CIS or early MS. Our findings encourage the application of both $\mathrm{sNfL}$ and retinal measurements for risk stratification in clinical routine and trials.

\section{Acknowledgment}

The authors thank Charlotte Bereuter, Susan Pikol, and Cynthia Kraut for excellent technical support, and Alice Schneider and Frank Konietschke from the Charité Institute for biometry and clinical epidemiology for statistical consulting.

\section{Study Funding}

This study was supported by NeuroCure Clinical Research Center (NCRC), funded by the Deutsche Forschungsgemeinschaft (DFG, German Research Foundation) under Germany's Excellence Strategy—EXC-2049—390688087 (to F.P.) and a research grant from Novartis Pharma GmbH Deutschland (to H.G.Z.). 


\section{Disclosure}

T.-Y. Lin and V. Vitkova report no disclosures relevant to the manuscript. S. Asseyer has received conference grant from Celgene and speaking honoraria from Bayer Healthcare, Roche, and Alexion. I.M. Serra reports no disclosures relevant to the manuscript. S. Motamedi is named as coinventor on patent applications for retinal optical coherence tomography image analysis, unrelated to this work. C. Chien has received research support from Novartis, unrelated to this project. M. Ditzhaus, A. Papadopoulou, P. Benkert, and J. Kuhle report no disclosures relevant to the manuscript. J. Bellmann-Strobl has received travel grants and speaking honoraria from Bayer Healthcare, Biogen Idec, Merck Serono, Sanofi Genzyme, Teva Pharmaceuticals, Roche, and Novartis, none of them related to this work. K. Ruprecht received research support from Novartis, Merck Serono, German Ministry of Education and Research, European Union (821283-2), Stiftung Charité (BIH Clinical Fellow Program), and Arthur Arnstein Foundation; received speaker honoraria and travel grants from Bayer, Biogen Idec, Merck Serono, Sanofi-Aventis/Genzyme, Teva, Roche, Novartis, and The Guthy-Jackson Charitable Foundation. F. Paul reports research grants and speaker honoraria from Bayer, Teva, Genzyme, Merck, Novartis, and MedImmune and is a member of the steering committee of the OCTIMS study (Novartis). A.U. Brandt is cofounder and shareholder of Motognosis and Nocturne; he is named as inventor on several patent applications regarding MS serum biomarkers, OCT image analysis, and perceptive visual computing. H.G. Zimmermann received research grants from Novartis Pharma GmbH Deutschland and speaking honoraria from Bayer Healthcare. Go to Neurology.org/NN for full disclosures.

\section{Publication History}

Received by Neurology: Neuroimmunology \& Neuroinflammation February 10, 2021. Accepted in final form May 10, 2021.

\section{Appendix Authors}

\begin{tabular}{|c|c|c|}
\hline Name & Location & Contribution \\
\hline Ting-Yi Lin, MD & $\begin{array}{l}\text { Charité-Universitätsmedizin } \\
\text { Berlin, Germany }\end{array}$ & $\begin{array}{l}\text { Conducted the } \\
\text { statistical analysis, } \\
\text { contributed to data } \\
\text { interpretation, and } \\
\text { drafted the manuscript } \\
\text { for intellectual content }\end{array}$ \\
\hline $\begin{array}{l}\text { Viktoriya Vitkova, } \\
\text { MSc }\end{array}$ & $\begin{array}{l}\text { Charité-Universitätsmedizin } \\
\text { Berlin, Germany }\end{array}$ & $\begin{array}{l}\text { Performed OCT quality } \\
\text { control and } \\
\text { segmentation and } \\
\text { revised the manuscript } \\
\text { for intellectual content }\end{array}$ \\
\hline $\begin{array}{l}\text { Susanna Asseyer, } \\
\text { MD }\end{array}$ & $\begin{array}{l}\text { Charité-Universitätsmedizin } \\
\text { Berlin, Germany }\end{array}$ & $\begin{array}{l}\text { Provided clinical data } \\
\text { and revised the } \\
\text { manuscript for } \\
\text { intellectual content }\end{array}$ \\
\hline $\begin{array}{l}\text { Ivette Martorell } \\
\text { Serra, MSc }\end{array}$ & $\begin{array}{l}\text { Charité-Universitätsmedizin } \\
\text { Berlin, Germany }\end{array}$ & $\begin{array}{l}\text { Performed OCT quality } \\
\text { control and } \\
\text { segmentation and } \\
\text { revised the manuscript } \\
\text { for intellectual content }\end{array}$ \\
\hline
\end{tabular}

Appendix (continued)

\begin{tabular}{|c|c|c|}
\hline Name & Location & Contribution \\
\hline $\begin{array}{l}\text { Seyedamirhosein } \\
\text { Motamedi, PhD }\end{array}$ & $\begin{array}{l}\text { Charité-Universitätsmedizin } \\
\text { Berlin, Germany }\end{array}$ & $\begin{array}{l}\text { Performed OCT quality } \\
\text { control and } \\
\text { segmentation and } \\
\text { revised the manuscript } \\
\text { for intellectual content }\end{array}$ \\
\hline $\begin{array}{l}\text { Claudia Chien, } \\
\text { PhD }\end{array}$ & $\begin{array}{l}\text { Charité-Universitätsmedizin } \\
\text { Berlin, Germany }\end{array}$ & $\begin{array}{l}\text { Analyzed MRI data and } \\
\text { revised the manuscript } \\
\text { for intellectual content }\end{array}$ \\
\hline $\begin{array}{l}\text { Marc Ditzhaus, } \\
\text { PhD }\end{array}$ & $\begin{array}{l}\text { TU Dortmund University, } \\
\text { Germany }\end{array}$ & $\begin{array}{l}\text { Contributed to } \\
\text { statistical analysis and } \\
\text { data interpretation } \\
\text { and revised the } \\
\text { manuscript for } \\
\text { intellectual content }\end{array}$ \\
\hline $\begin{array}{l}\text { Athina } \\
\text { Papadopoulou, } \\
\text { MD }\end{array}$ & $\begin{array}{l}\text { University Hospital Basel, } \\
\text { Switzerland }\end{array}$ & $\begin{array}{l}\text { Contributed to data } \\
\text { interpretation and } \\
\text { revised the manuscript } \\
\text { for intellectual content }\end{array}$ \\
\hline $\begin{array}{l}\text { Pascal Benkert, } \\
\text { PhD }\end{array}$ & $\begin{array}{l}\text { University Hospital Basel, } \\
\text { Switzerland }\end{array}$ & $\begin{array}{l}\text { Provided sNfL analysis } \\
\text { and revised the } \\
\text { manuscript for } \\
\text { intellectual content }\end{array}$ \\
\hline Jens Kuhle, MD & $\begin{array}{l}\text { University Hospital Basel, } \\
\text { Switzerland }\end{array}$ & $\begin{array}{l}\text { Conceived the study, } \\
\text { provided sNfL analysis, } \\
\text { contributed to data } \\
\text { interpretation, and } \\
\text { revised the manuscript } \\
\text { for intellectual content }\end{array}$ \\
\hline $\begin{array}{l}\text { Judith Bellmann- } \\
\text { Strobl, MD }\end{array}$ & $\begin{array}{l}\text { Charité-Universitätsmedizin } \\
\text { Berlin, Germany }\end{array}$ & $\begin{array}{l}\text { Provided clinical data } \\
\text { and revised the } \\
\text { manuscript for } \\
\text { intellectual content }\end{array}$ \\
\hline $\begin{array}{l}\text { Klemens } \\
\text { Ruprecht, MD }\end{array}$ & $\begin{array}{l}\text { Charité-Universitätsmedizin } \\
\text { Berlin, Germany }\end{array}$ & $\begin{array}{l}\text { Conceived the study, } \\
\text { contributed to data } \\
\text { interpretation, and } \\
\text { revised the manuscript } \\
\text { for intellectual content }\end{array}$ \\
\hline $\begin{array}{l}\text { Friedemann Paul, } \\
\text { MD }\end{array}$ & $\begin{array}{l}\text { Charité-Universitätsmedizin } \\
\text { Berlin, Germany }\end{array}$ & $\begin{array}{l}\text { Conceived the study, } \\
\text { provided clinical data, } \\
\text { and revised the } \\
\text { manuscript for } \\
\text { intellectual content }\end{array}$ \\
\hline $\begin{array}{l}\text { Alexander U. } \\
\text { Brandt, MD }\end{array}$ & $\begin{array}{l}\text { Charité-Universitätsmedizin } \\
\text { Berlin, Germany }\end{array}$ & $\begin{array}{l}\text { Conceived the study, } \\
\text { contributed to data } \\
\text { interpretation, and } \\
\text { revised the manuscript } \\
\text { for intellectual content }\end{array}$ \\
\hline $\begin{array}{l}\text { Hanna G. } \\
\text { Zimmermann, } \\
\text { PhD }\end{array}$ & $\begin{array}{l}\text { Charité-Universitätsmedizin } \\
\text { Berlin, Germany }\end{array}$ & $\begin{array}{l}\text { Conceived the study, } \\
\text { supervised the } \\
\text { statistical analysis, } \\
\text { contributed to data } \\
\text { interpretation, and } \\
\text { drafted the manuscript } \\
\text { for intellectual content }\end{array}$ \\
\hline
\end{tabular}

\section{References}

1. Krieger SC, Cook K, de Nino S, Fletcher M. The topographical model of multiple sclerosis: a dynamic visualization of disease course. Neurol Neuroimmunol Neuroinflammation. 2016;3(5):e279.

2. Thompson AJ, Banwell BL, Barkhof F, et al. Diagnosis of multiple sclerosis: 2017 revisions of the McDonald criteria. Lancet Neurol. 2018;17(2):162-173.

3. Reich DS, Lucchinetti CF, Calabresi PA. Multiple slcerosis. N Engl J Med. 2018; 378(2):169-180.

4. Oberwahrenbrock T, Schippling S, Ringelstein M, et al. Retinal damage in multiple sclerosis disease subtypes measured by high-resolution optical coherence tomography. Mult Scler Int. 2012;2012:530305. 
5. Zimmermann H, Oberwahrenbrock T, Brandt AU, Paul F, Dörr JM. Optical coherence tomography for retinal imaging in multiple sclerosis. Degener Neurol Neuromuscul Dis. 2014;4:153-162.

6. Balk LJ, Cruz-Herranz A, Albrecht $P$, et al. Timing of retinal neuronal and axonal loss in MS: a longitudinal OCT study. J Neurol. 2016;263(7):1323-1331.

7. Oberwahrenbrock T, Traber GL, Lukas S, et al. Multicenter reliability of semiautomatic retinal layer segmentation using OCT. Neurol Neuroimmunol Neuroinflammation. 2018;5(3):e449.

8. Zimmermann HG, Knier B, Oberwahrenbrock T, et al. Association of retinal ganglion cell layer thickness with future disease activity in patients with clinically isolated syndrome. JAMA Neurol. 2018;75(9):1071-1079.

9. Barro C, Benkert P, Disanto G, et al. Serum neurofilament as a predictor of disease worsening and brain and spinal cord atrophy in multiple sclerosis. Brain. 2018;141(8): 2382-2391.

10. Bittner S, Steffen F, Uphaus T, et al. Clinical implications of serum neurofilament in newly diagnosed MS patients: a longitudinal multicentre cohort study. EBioMedicine. 2020;56:102807.

11. Disanto G, Barro C, Benkert P, et al. Serum neurofilament light: a biomarker of neuronal damage in multiple sclerosis. Ann Neurol. 2017;81(6):857-870.

12. Kuhle J, Kropshofer H, Haering DA, et al. Blood neurofilament light chain as a biomarker of MS disease activity and treatment response. Neurology. 2019;92(10): e1007-e1015.

13. Siller N, Kuhle J, Muthuraman M, et al. Serum neurofilament light chain is a biomarker of acute and chronic neuronal damage in early multiple sclerosis. Mult Scler J. 2019;25(5):678-686.

14. Saraste M, Bezukladova S, Matilainen M, et al. High serum neurofilament associates with diffuse white matter damage in MS. Neurol Neuroimmunol Neuroinflammation. 2020;8(1):e926.

15. Thebault S, Abdoli M, Fereshtehnejad SM, et al. Serum neurofilament light chain predicts long term clinical outcomes in multiple sclerosis. Sci Rep. 2020;10(1):10381.

16. Rosso M, Gonzalez CT, Healy BC, et al. Temporal association of sNfL and gadenhancing lesions in multiple sclerosis. Ann Clin Transl Neurol. 2020;7(6):945-955

17. Rotstein DL, Healy BC, Malik MT, Chitnis T, Weiner HL. Evaluation of no evidence of disease activity in a 7-year longitudinal multiple sclerosis cohort. JAMA Neurol. 2015;72(2):152-158.

18. Kalincik T, Cutter G, Spelman T, et al. Defining reliable disability outcomes in multiple sclerosis. Brain. 2015;138(pt 11):3287-3298.

19. Kuhle J, Barro C, Andreasson U, et al. Comparison of three analytical platforms for quantification of the neurofilament light chain in blood samples: ELISA, electrochemiluminescence immunoassay and Simoa. Clin Chem Lab Med. 2016;54(10): 1655-1661.

20. Tewarie P, Balk L, Costello F, et al. The OSCAR-IB consensus criteria for retinal OCT quality assessment. PLoS One. 2012;7(4):e34823.

21. Schippling S, Balk LJ, Costello F, et al. Quality control for retinal OCT in multiple sclerosis: validation of the OSCAR-IB criteria. Mult Scler J. 2015;21(2):163-170.

22. Cruz-Herranz A, Balk LJ, Oberwahrenbrock T, et al. The APOSTEL recommendations for reporting quantitative optical coherence tomography studies. Neurology. 2016;86(24):2303-2309.

23. Motamedi S, Gawlik K, Ayadi N, et al. Normative data and minimally detectable change for inner retinal layer thicknesses using a semi-automated OCT image segmentation pipeline. Front Neurol. 2019;10:1117.
24. Oberwahrenbrock T, Weinhold M, Mikolajczak J, et al. Reliability of intra-retinal layer thickness estimates. PLoS One. 2015;10(9):e0137316.

25. Jenkinson M, Smith S. A global optimisation method for robust affine registration of brain images. Med Image Anal. 2001;5(2):143-156.

26. Jenkinson M, Bannister P, Brady M, Smith S. Improved optimization for the robust and accurate linear registration and motion correction of brain images. Neuroimage. 2002;17(2):825-841.

27. Yushkevich PA, Piven J, Hazlett HC, et al. User-guided 3D active contour segmentation of anatomical structures: significantly improved efficiency and reliability. Neuroimage. 2006;31(3):1116-1128.

28. R Core Team. R: A Language and Environment for Statistical Computing [online]. R Foundation for Statistical Computing; 2018. Accessed June 25, 2020. Available at: R-project.org/.

29. Tintore M, Rovira A, Río J, et al. Defining high, medium and low impact prognostic factors for developing multiple sclerosis. Brain. 2015;138(pt 7):1863-1874.

30. Li R, Chambless L. Test for additive interaction in proportional hazards models. Ann Epidemiol. 2007;17(3):227-236.

31. Bsteh G, Berek K, Hegen H, et al. Serum neurofilament levels correlate with retinal nerve fiber layer thinning in multiple sclerosis. Mult Scler J. 2020;26(13):1682-1690.

32. Tavazzi E, Jakimovski D, Kuhle J, et al. Serum neurofilament light chain and optical coherence tomography measures in MS: a longitudinal study. Neurol Neuroimmuno Neuroinflammation. 2020;7(4):e737.

33. Kuhle J, Disanto G, Dobson R, et al. Conversion from clinically isolated syndrome to multiple sclerosis: a large multicentre study. Mult Scler. 2015;21(8):1013-1024.

34. Bridel C, Van Wieringen WN, Zetterberg H, et al. Diagnostic value of cerebrospinal fluid neurofilament light protein in neurology: a systematic review and meta-analysis. JAMA Neurol. 2019;76(9):1035-1048.

35. Barro C, Chitnis T, Weiner HL. Blood neurofilament light: a critical review of its application to neurologic disease. Ann Clin Transl Neurol. 2020;7(12):2508-2523.

36. Balk LJ, Coric D, Knier B, et al. Retinal inner nuclear layer volume reflects inflammatory disease activity in multiple sclerosis; a longitudinal OCT study. Mult Scler J Exp Transl Clin. 2019;5(3):2055217319871582.

37. Knier B, Schmidt P, Aly L, et al. Retinal inner nuclear layer volume reflects response to immunotherapy in multiple sclerosis. Brain. 2016;139(11):2855-2863.

38. Petzold A, Wattjes MP, Costello F, et al. The investigation of acute optic neuritis: a review and proposed protocol. Nat Rev Neurol. 2014;10(8):447-458.

39. Britze J, Pihl-Jensen G, Frederiksen JL. Retinal ganglion cell analysis in multiple sclerosis and optic neuritis: a systematic review and meta-analysis. J Neurol. 2017, 264(9):1837-1853.

40. Gabilondo I, Martínez-Lapiscina EH, Fraga-Pumar E, et al. Dynamics of retinal injury after acute optic neuritis. Ann Neurol. 2015;77(3):517-528.

41. Knier B, Leppenetier G, Wetzlmair C, et al. Association of retinal architecture, intrathecal immunity, and clinical course in multiple sclerosis. JAMA Neurol. 2017;74(7):847-856.

42. Gattringer T, Pinter D, Enzinger C, et al. Serum neurofilament light is sensitive to active cerebral small vessel disease. Neurology. 2017;89(5):2108-2114.

43. Korley FK, Goldstick J, Mastali M, et al. Serum NfL (neurofilament light chain) levels and incident stroke in adults with diabetes mellitus. Stroke. 2019;50(7):1669-1675.

44. Manouchehrinia A, Piehl F, Hillert J, et al. Confounding effect of blood volume and body mass index on blood neurofilament light chain levels. Ann Clin Transl Neurol. 2020;7(1):139-143.

45. Verduyn C, Bjerke M, Duerinck J, et al. CSF and blood neurofilament levels in athletes participating in physical contact sports: a systematic review. Neurology. 2021;96(15):705-715 


\title{
Neurology \\ Neuroimmunology \& Neuroinflammation
}

\author{
Increased Serum Neurofilament Light and Thin Ganglion Cell-Inner Plexiform Layer \\ Are Additive Risk Factors for Disease Activity in Early Multiple Sclerosis \\ Ting-Yi Lin, Viktoriya Vitkova, Susanna Asseyer, et al. \\ Neurol Neuroimmunol Neuroinflamm 2021;8; \\ DOI 10.1212/NXI.0000000000001051
}

This information is current as of August 4, 2021

Updated Information \&
Services
References
Citations
Subspecialty Collections

Permissions \& Licensing

Reprints including high resolution figures, can be found at:

http://nn.neurology.org/content/8/5/e1051.full.html

This article cites 44 articles, 0 of which you can access for free at: http://nn.neurology.org/content/8/5/e1051.full.html\#\#ref-list-1

This article has been cited by 1 HighWire-hosted articles: http://nn.neurology.org/content/8/5/e1051.full.html\#\#otherarticles

This article, along with others on similar topics, appears in the following collection(s):

Multiple sclerosis

http://nn.neurology.org//cgi/collection/multiple_sclerosis

\section{Retina}

http://nn.neurology.org//cgi/collection/retina

Information about reproducing this article in parts (figures,tables) or in its entirety can be found online at:

http://nn.neurology.org/misc/about.xhtml\#permissions

Information about ordering reprints can be found online:

http://nn.neurology.org/misc/addir.xhtml\#reprintsus

Neurol Neuroimmunol Neuroinflamm is an official journal of the American Academy of Neurology.

Published since April 2014, it is an open-access, online-only, continuous publication journal. Copyright

Copyright $\odot 2021$ The Author(s). Published by Wolters Kluwer Health, Inc. on behalf of the American

Academy of Neurology.. All rights reserved. Online ISSN: 2332-7812.

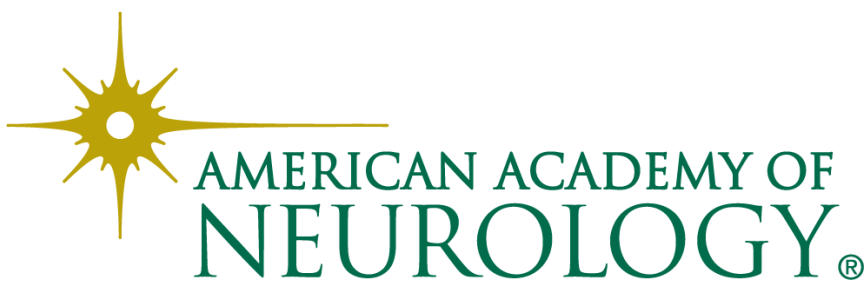

